\title{
Taking the "Pulse" of Pulsed Field Ablation: Real-World Experience
}

\author{
Tina Baykaner ${ }^{1}$, Muhammad Fazal ${ }^{2}$, and Atul Verma ${ }^{3}$ \\ ${ }^{1}$ Stanford Medicine \\ ${ }^{2}$ Stanford University \\ ${ }^{3}$ Southlake Regional Health Centre
}

December 4, 2021

Title:Taking the "Pulse" of Pulsed Field Ablation: Real-World Experience

Authors:

Tina Baykaner, MD, $\mathrm{MPH}^{1}$; Muhammad Fazal, $\mathrm{MD}^{1}$ and Atul Verma, MD ${ }^{2}$

${ }^{1}$ Stanford University, Stanford, CA, USA

${ }^{2}$ Southlake Regional Health Centre, University of Toronto, Toronto, CA

\section{Correspondence:}

Tina Baykaner, MD MPH, 453 Quarry Road, 334C, Stanford CA 94304

Atul Verma, MD, 596 Davis Dr, Newmarket, ON L3Y 2P9, Canada

Voice: +1 650-7362563 Fax: +1 650-7246131;Email: tina4@stanford.edu; atul.verma@utoronto.ca

Word Count: 1365 excluding title page and references

Keywords: Atrial Fibrillation, Catheter Ablation, Pulsed Field Ablation

Conflicts of Interest and Funding: Dr. Baykaner reports grant support from National Institutes of Health (HL145017) and serves in the advisory boards of Medtronic, BIOTRONIK and PaceMate. Dr. Fazal reports no disclosures. Dr. Verma reports grant support from Medtronic, Biosense Webster, Abbott and Bayer and serves in the advisory boards of Medtronic, Biosense Webster, Boston Scientific, Adagio, Ablacon, Kardium and Galaxy Medical.

Atrial fibrillation (AF) ablation is the cornerstone of therapy for symptomatic AF, with now increasing data on the safety and efficacy of this approach over medical management in improving quality of life, decreasing rates of stroke, cardiac hospitalizations and providing mortality benefit in several populations [1]. Cardiac ablation using pulsed field ablation (PFA) provides an exciting non-thermal approach, enabling ablation lesions without causing thermal collateral injury to structures such as the esophagus or phrenic nerve.

Multiple in-human studies since 2018 have shown feasibility and safety of pulmonary vein isolation (PVI) with PFA in patients with paroxysmal AF. Reddy et al. using a basket/flower over the wire ablation catheter, a custom-built generator and monophasic bipolar PFA waveforms were able to achieve $96 \%$ durable PVI at 3-months with 1-year freedom from $\mathrm{AF}$ of $84.5 \%$ [2]. Another circular catheter-based system delivering bipolar, biphasic waveforms have also demonstrated excellent acute procedural outcomes with no significant procedural complications [3]. The only other PFA system tested in patients with published acute outcomes involves 10 patients with paroxysmal and persistent AF, showing the feasibility and safety of acute PVI.[4] 
PFA may also be quite effective for lesions outside the pulmonary veins (PVs). Reddy et al. demonstrated $100 \%$ durability of both posterior wall isolation and cavotricuspid isthmus (CTI) line at the time of 3-month remapping in 25 patients with persistent AF [5]. Moreover, PFA with a novel lattice tip catheter was able to achieve $100 \%$ acute success in achieving mitral $(n=14)$, roof $(n=34)$ and CTI lines $(n=44)$ in a mixed cohort of paroxysmal and persistent AF patients [6]. Verma et al. also demonstrated acute posterior wall isolation in an extended pilot study of a circular-based PFA system [3]. However, all of these studies were performed by a limited number of investigators in a limited number of patients. Now that PFA is approved in the European Union (EU), publications of "real-world" experiences are needed.

In this issue of the Journal of Cardiovascular Electrophysiology,Gunawardene et al. studied PFA combined with ultra-high-density mapping in 20 patients with paroxysmal or persistent AF undergoing first time catheter ablation in a prospective, single arm, observational study. All patients underwent PVI, and nine patients with persistent $\mathrm{AF}$ also underwent additional ablation including left atrial posterior wall isolation and mitral isthmus ablation using a multispline PFA catheter (FARAWAVE, Boston Scientific, MA, USA). The authors also utilized an electroanatomical mapping system (Rhythmia ${ }^{\mathrm{TM}}$, Boston Scientific, MA, USA) to perform voltage mapping and localize the PFA catheter. Using an initial generator output of $1900 \mathrm{~V}$, PVI was performed in all patients using at least 8 applications (4 basket, 4 flower). If post-ablation voltage mapping found conduction gaps or PV reconnections, additional ablations were performed with $2000 \mathrm{~V}$.

The authors confirmed several important assumptions about PFA. First, they found a very high rate of acute PV and extra-PV isolation (posterior wall, mitral isthmus) with rapid and significant abatement of electrogram voltage over a large atrial surface area. The lesions also seemed to have "smooth", welldemarcated borders as promised by the preclinical studies [7]. PFA created wide antral circumferential lesions with no complex fractionated electrograms (CFAE) along the PV ostia in $85.2 \%$ of patients and none on the LA posterior wall in $62.5 \%$ of patients. The authors also showed that PFA could be performed with very few major complications. No esophageal lesions were seen on endoscopy and no phrenic injury occurred.

Importantly, they also showed that PFA could be integrated with an electroanatomical mapping system. Current electroanatomical mapping systems use a combination of impedance based tracking through skin surface patches and magnetic tracking using localization pads. Some even run a small electrical current through the surface patches. These systems were never designed to deal with delivery of large voltages to the patient for ablation. These large voltages acutely change the impedance of the catheter tip and possibly even the skin patches (depending on whether energy delivery is bipolar or unipolar). This will likely affect visualization of the catheter during PFA delivery and also the stability of the map for the duration of the case. The authors, for example, did not mention if the catheter disappeared from view during delivery of the electrical fields and then re-appeared. The PFA catheter was not visualized in a minority of patients $(10 \%)$, and the location of the catheter did not match with the level of isolation in $12 \%$ of patients for right PVs. Map shift did occur in one patient (5\%). The catheter also could not be visualized in its different shapes and a work-around was created such that the catheter appeared as a circle. These problems can be overcome with hardware and software adjustments and manufacturers have already shown that successful integration can occur $[8,9]$. This is critical since the reported left atrial dwell time for the patients was less than one hour, but fluoroscopy time was over 19 minutes. The full promise of PFA's efficiency will need to be realized in conjunction with successful integration with electroanatomic mapping.

Interestingly the authors found five early PV reconnections $(5 / 80,6.25 \%$ acute reconnection rate) in postablation mapping. All the connection gaps were located at the anterior superior aspect of the PV ostium. Yet rapid and significant electrogram abatement was seen with the initial delivery of PFA. This is one of the fundamental issues with current PFA delivery systems. The pulses have been designed and optimized to create "transmural" lesions in pre-clinical models and confirmed with some early human remapping studies. However, PFA acutely stuns the electrical activity of cardiac cells. Even a sub-therapeutic, single delivery of PFA can cause electrograms to disappear. Furthermore, data has shown that repetitive applications [10] are required to "push" the depth of the lesion to its maximum - explaining the 8 pre-determined applications that were delivered per site in this manuscript. But how does an operator assess when enough deliveries have been 
placed? Do we need to have waiting periods of 20 minutes or longer? Would this study have found higher rates of reconnection if this had been done? Are there other electrogram or procedural components that need to be monitored? This study importantly raises all of these questions which will need to be answered.

The study also alludes to some other unknowns about PFA. They reported one case of coronary artery spasm which fortunately resolved rapidly with administration of nitroglycerin. Many studies have shown that PFA preserves both microvasculature in the tissue and the integrity of larger vessels [11,12]. It is therefore unlikely that permanent coronary damage will occur. However, ablation in the mitral and cavotricuspid isthmuses are close to coronary vessels and operators must be conscious of the possibility of spasm. Pronounced vagal responses have also been reported by several PFA systems $[2,8]$. This is likely due to partial stimulation and/or ablation of the autonomic ganglionated plexi. Unlike myelinated nerves which are insulated against PFA, the cell bodies of plexi are prone to PFA ablation [8]. The authors have shown nicely that pre-ablation atropine or pacing can easily resolve this issue and it would be interesting to know whether additional ablation of sites of ganglionated plexi will add to procedural success.

Finally, the biggest limitation of currently evaluated PFA systems is tissue depth. Although there is excitement about the possibility of greater transmurality with PFA, tissue depth of current PFA systems may be limited to the standard 3-5 mm, also achievable by thermal ablation. Hence, it is not surprising that acute reconnection was seen in this study. The study also used PFA on the mitral isthmus which can be relatively thick $(0.6-1.4 \mathrm{~cm})$. Although they were able to achieve acute block across the mitral isthmus, it is far from clear that this block will be permanent. Adjustments are being made to achieve deeper lesions with PFA, with the potential for creating adverse thermal effects and skeletal muscle contraction[13,14].

In summary, the authors should be congratulated on providing some of the first "real world" evidence on PFA for AF ablation and integration with mapping. It is reports like this that will help us to keep the "pulse" of PFA in the years to come.

\section{References:}

1. Andrade JG, Wells GA, Deyell MW, Bennett M, Essebag V, Champagne J, et al. Cryoablation or Drug Therapy for Initial Treatment of Atrial Fibrillation. N Engl J Med. 2021;384: 305-315. doi:10.1056/NEJMoa2029980

2. Reddy VY, Dukkipati SR, Neuzil P, Anic A, Petru J, Funasako M, et al. Pulsed Field Ablation of Paroxysmal Atrial Fibrillation: 1-Year Outcomes of IMPULSE, PEFCAT, and PEFCAT II. JACC Clin Electrophysiol. 2021;7: 614-627. doi:10.1016/j.jacep.2021.02.014

3. Verma A, Boersma LV, Hummel JD, Calkins H, Sanders P, Haines DE, et al. PULSED AF: first human experience and acute procedural outcomes using a novel pulsed field ablation system. Heart Rhythm. 2020;8.

4. Loh P, van Es R, Groen MHA, Neven K, Kassenberg W, Wittkampf FHM, et al. Pulmonary Vein Isolation With Single Pulse Irreversible Electroporation: A First in Human Study in 10 Patients With Atrial Fibrillation. Circ Arrhythm Electrophysiol. 2020;13: e008192. doi:10.1161/CIRCEP.119.008192

5. Reddy VY, Anic A, Koruth J, Petru J, Funasako M, Minami K, et al. Pulsed Field Ablation in Patients With Persistent Atrial Fibrillation. J Am Coll Cardiol. 2020;76: 1068-1080. doi:10.1016/j.jacc.2020.07.007

6. Reddy VY, Anter E, Rackauskas G, Peichl P, Koruth JS, Petru J, et al. Lattice-Tip Focal Ablation Catheter That Toggles Between Radiofrequency and Pulsed Field Energy to Treat Atrial Fibrillation. Circulation: Arrhythmia and Electrophysiology. 2020. doi:10.1161/circep.120.008718

7. Stewart MT, Haines DE, Verma A, Kirchhof N, Barka N, Grassl E, et al. Intracardiac pulsed field ablation: Proof of feasibility in a chronic porcine model. Heart Rhythm. 2019;16: 754-764. doi:10.1016/j.hrthm.2018.10.030

8. Verma A, Asivatham SJ, Deneke T, Castellvi Q, Neal RE. Primer on Pulsed Electrical Field Ablation. Circ Arrhythm Electrophysiol. 2021;14: e010086. doi:10.1161/CIRCEP.121.010086 
9. Nakagawa H, Castellvi Q, Neal R, Girouard S, Kuroda S, Hussein AA, et al. B-PO02-133 HISTOLOGICAL CHARACTERIZATION OF REVERSIBLE AND IRREVERSIBLE VENTRICULAR LESION BOUNDARIES PRODUCED BY PULSED FIELD ABLATION. Heart Rhythm. 2021;18: S151-S152. doi:10.1016/j.hrthm.2021.06.386

10. Yavin HD, Higuchi K, Sroubek J, Younis A, Zilberman I, Anter E. Pulsed-Field Ablation in Ventricular Myocardium Using a Focal Catheter. Circ Arrhythm Electrophysiol. 2021;14: e010375. doi:10.1161/CIRCEP.121.010375

11. van Driel VJHM, Neven KGEJ, van Wessel H, du Pré BC, Vink A, Doevendans PAFM, et al. Pulmonary vein stenosis after catheter ablation: electroporation versus radiofrequency. Circ Arrhythm Electrophysiol. 2014;7: 734-738. doi:10.1161/CIRCEP.113.001111

12. Stewart MT, Haines DE, Miklavčič D, Kos B, Kirchhof N, Barka N, et al. Safety and chronic lesion characterization of pulsed field ablation in a Porcine model. J Cardiovasc Electrophysiol. 2021;32: 958-969. doi: $10.1111 /$ jce. 14980

13. van Zyl M, Ladas TP, Yasin OZ, Ladejobi AO, Tan NYL, Christopoulos G, et al. B-PO03-028 BIPOLAR PULSED ELECTRIC FIELD ABLATION OF THE INTERVENTRICULAR SEPTUM. Heart Rhythm. 2021;18: S200. doi:10.1016/j.hrthm.2021.06.504

14. Anter E, Yavin H, Biton D, Sroubek J, Higuchi K, Saliba WI. B-PO02-131 VENTRICULAR ABLATION USING PULSED-FIELD ENERGY WITH A LATTICE-TIP CATHETER. Heart Rhythm. 2021;18: S151. doi:10.1016/j.hrthm.2021.06.384 